\title{
Location-based fatigue damage assessment on an FPSO by the spectral analysis method
}

\author{
$A$ Viswanathan*, $V A$ Subramanian, and $D$ Kumar \\ Department of Ocean Engineering, Indian Institute of Technology Madras, Chennai -600036, India
}

\begin{abstract}
Floating production storage and offloading ships (FPSOs) are offshore platforms positioned at a location and operating at different loading conditions and corresponding draughts. Due to their static location, they get subjected to continuous fluctuating loads due to the sea environment. For these reasons the fatigue damage prediction of an FPSO is essential. The emphasis of this study is on the effect of fatigue damage at critical joints of critical structural members under the influence of vertical and horizontal bending in isolation and combination. The method considers an FPSO of $320 \mathrm{~m}$ length for a case study, selecting a total of fourteen such structural member locations along the periphery at its midship section. The analysis considers two necessary loading conditions namely, fully loaded and ballast condition. The study uses spectral fatigue analysis method wherein the bending moment transfer function generates the stress transfer function, which in turn helps to generate the power spectral density function. Combining the spectral moments with Palmgren-Miner rule gives the cumulative fatigue damage of the FPSO. The results provide an insight into the variation in fatigue damage concerning the location of the structural member under the influence of vertical and horizontal bending moments.
\end{abstract}

\section{Introduction}

Floating production storage and offloading ships (FPSOs) are offshore installations deployed for oil production, storage, and offloading, and provided with living facilities and power generation modules. They are continuously subjected to wind and sea wave forces and are prone to fatigue. Fatigue life estimation of FPSO under service loading is an essential criterion for both safe and economic design and operation. The fatigue life of a vessel can be estimated either by a simplified deterministic method such as IACS R 56 (Recommendation of the International Association of Classified Societies) or by spectraltechnique. Spectral fatigue analysis provides a reliable technique to analyse different sea states along with their probability of occurrence. Initially, fatigue damage estimation for plated structures used the nominal stress concept [1]. The method of spectral fatigue analysis for offshore structures was introduced in 1976 [2]. This method has become a standard in the fatigue analysis approach for offshore structures [3]. Since ships get

\footnotetext{
* Corresponding author: arjunviswanathan@outlook.com
} 
frequently exposed to vertical and horizontal bending moments due to the ocean waves, their combined effect comes into consideration in the fatigue damage estimation using the spectral technique [4]. The spectral analysis taking into consideration waves as the solitary fluctuating load, revealed significant uncertainties in studies conducted by Classification societies [5] Further, the hot spot technique was then combined with various enhancements of spectral technique by the classification societies [6] [7] and included in guidelines for fatigue assessment of ship structures [8]. In 2010, studies were conducted to analyse the effect of spectral width parameter in combination with the bandwidth parameter, to obtain the spectral fatigue damage calculation of ships [9].

Fatigue damage of any structural member is directly related to its location. Since the primary source of fatigue is due to the action of waves, the force mainly induced on the structure in the form of vertical and horizontal bending moments. Assessment of fatigue damage requires a detailed study about the influence of structural member location. For proper design, considering fatigue, it is also essential to assess the effect of vertical and horizontal bending moments. This paper considers the influence of vertical and horizontal bending moments as well as structural member location for fatigue damage. Vertical and horizontal bending moment transfer functions considered in isolation and combination, concerning the geometrical parameters, give the stress transfer function. This study uses ANSYS AQWA to obtain the vertical and horizontal bending moment transfer functions for different heading angles. A MATLAB code computes the stress transfer functions and predicts the fatigue damage using the spectral technique.

\section{Methodology and theoretical background}

The methodology for the estimation of fatigue damage by spectral technique involves the following five significant steps, see figure 1.

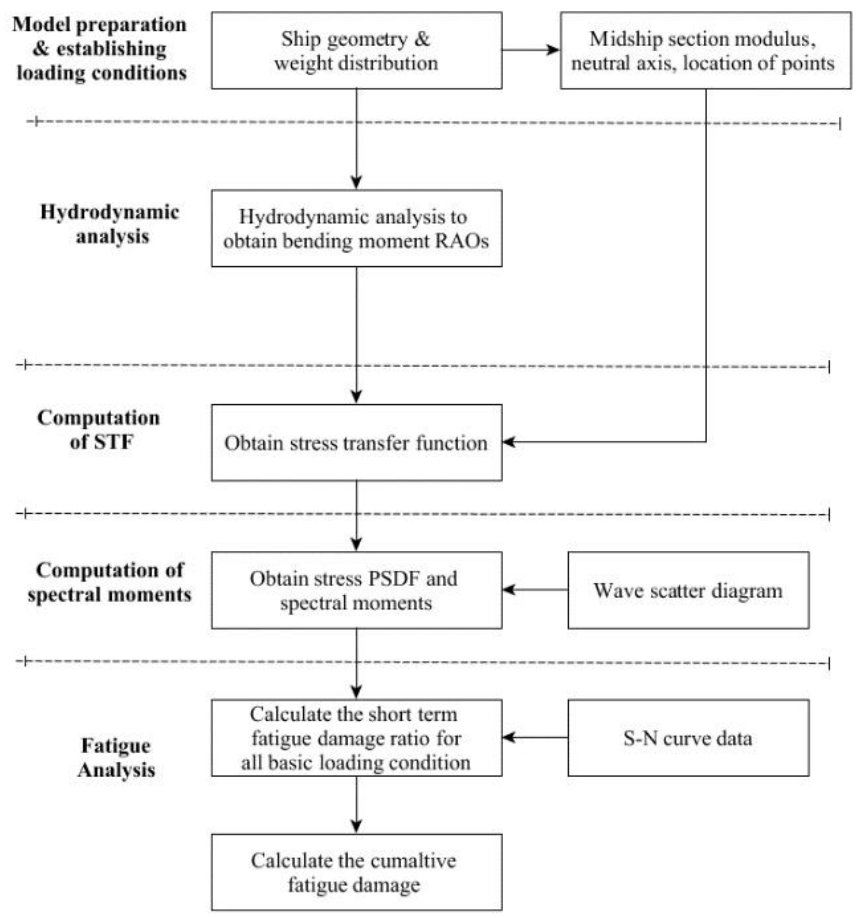

Fig. 1. The applied methodology for spectral fatigue analysis. 


\subsection{Hydrodynamic model preparation \& establishing the loading conditions}

\subsubsection{Hydrodynamic model preparation}

Table 1 gives the particulars of the ship taken up for investigation here. The 3D hull shape geometry along with the distribution of weight along the length of the ship form the basis for preparation of the hydrodynamic model. The model prepares the load and consequent vertical and horizontal bending moment components distribution on the hull. At this stage, input the mass moment of inertia/radius of gyration for the sections along the length.

Table 1. Ship particulars.

\begin{tabular}{|c|c|}
\hline \multicolumn{2}{|c|}{ Ship Dimensions } \\
\hline Length Overall & $320 \mathrm{~m}$ \\
\hline Length between perpendicular & $312 \mathrm{~m}$ \\
\hline Moulded Breadth & 45.33 \\
\hline Moulded Depth & $30 \mathrm{~m}$ \\
\hline Deepest draught & $20 \mathrm{~m}$ \\
\hline Shallowest draught & $16 \mathrm{~m}$ \\
\hline Displacement (Fully loaded) & $275465 \mathrm{~T}$ \\
\hline Displacement (Ballast) & $218438 \mathrm{~T}$ \\
\hline Midship section properties \\
\hline The distance of the neutral axis from the baseline \\
\hline Midship section moment of inertia to the z-axis & $12.33 \mathrm{~m}$ \\
\hline Midship section moment of inertia to the y-axis & $2255 \mathrm{~m}^{4}$ \\
\hline
\end{tabular}

\subsubsection{Loading conditions}

Change of draught, internal loading condition and external waves can induce fluctuating load conditions on the FPSO during its lifetime operations. The spectral method here considers two basic loading conditions of the FPSO as a minimum requirement namely, the most in-depth and shallowest draught condition during the on-site service life.

The method computes the fatigue damage and expected fatigue life for the FPSO vessel for each loading condition. The weighted average of the reciprocals of individual fatigue life computes 'combined fatigue life'. If each loading condition has an exposure time factor of 50 per cent each, then the combined fatigue life Lc is obtained as [10]:

$$
L_{C}=\left(\frac{0.5}{L_{1}}+\frac{0.5}{L_{2}}\right)^{-1}
$$

where L1 and L2 are the computed fatigue lives for the structural member in a specific location during the respective base loading conditions.

\subsubsection{Hydrodynamic analysis for load computation in regular waves}

The lightweight, deadweight and centre of gravity information are inputs for calculation of the weight distribution of the FPSO at both the loading conditions. Application of the commercial code ANSYS AQWA gives the bending moment response amplitude operator (RAO) which is the bending moment for unit wave amplitude, for all loading conditions. The notations $\mathrm{RAO}_{\mathrm{M}, \mathrm{v}}$ and $\mathrm{RAO}_{\mathrm{M}, \mathrm{h}}$ denote the vertical and horizontal component RAOs respectively at the midship section. 


\subsection{Computation of stress transfer function (STF)}

The corresponding bending stress RAOs namely, $\mathrm{RAO}_{\sigma, \mathrm{v}}$ and $\mathrm{RAO}_{\sigma, \mathrm{h}}$ follow easily from knowledge of the area moments of inertia $I_{y y}$ and $I_{z z}$ and the distances of the sections from the neutral axes. From these results the new combined stress RAO [11]. The equations are as follows:

$$
\begin{gathered}
R A O_{\sigma, v}=\frac{z-z_{0}}{I_{y y}} R A O_{M, v} \\
R A O_{\sigma, h}=\frac{y}{I_{z z}} R A O_{M, h}
\end{gathered}
$$

Here $\mathrm{z}$ and $\mathrm{z}_{0}$ are the vertical distances from the baseline to the structural member location and the neutral axis, respectively and $y$ is the horizontal distance of the structural member from the longitudinal centre plane.

The newly combined stress RAO now emerges as:

$$
R A O_{\sigma, c}=\left[\left(R A O_{\sigma, v}\right)^{2}+\left(R A O_{\sigma, h}\right)^{2}+\left(2 R A O_{\sigma, v} R A O_{\sigma, h} \cos \left(\varepsilon_{v}-\varepsilon_{h}\right)\right)\right]^{1 / 2}
$$

where $\varepsilon_{\mathrm{V}}$ and $\varepsilon_{\mathrm{h}}$ denote the phase of occurrence of the stress process due to vertical hull girder bending and the horizontal hull girder bending, respectively. Generate the stress transfer function from the total bending stress $\mathrm{RAO}_{\sigma, \mathrm{c}}$ as follows:

$$
\left|H_{\sigma}(\omega \mid \theta)\right|^{2}=\left(R A O_{\sigma, c}\right)^{2}
$$

where $\omega$ denotes the wave frequency, and $\theta$ is the heading angle.

\subsection{Computation of stress-energy spectrum \& spectral moments}

The FPSO experiences fluctuating loads mainly due to the incessantly changing waves around it during the service life, which causes fatigue damage to the structure. Divide the waves into different sea states using short-term wave statistics to describe each sea state. Use of wave scatter diagram permits to demarcate the probability of occurrence of sea state for long-term wave statistics. Pierson-Moskowitz (P-M) spectrum which is relevant for fully developed open ocean conditions is used and defined for different sea states as follows: [10]

$$
S_{\eta}\left(\omega \mid H_{s}, T_{z}\right)=\frac{H_{s}^{2}}{4 \pi \omega^{5}}\left(\frac{2 \pi}{T_{z}}\right)^{4} \exp \left[-\frac{1}{\pi}\left(\frac{2 \pi}{T_{z}}\right)^{4} \omega^{-4}\right]
$$

where $T_{z}$ is the zero crossing period, and $H_{s}$ is the significant wave height. The wave energy spectrum scales up with the stress transfer function to generate the stress-energy spectrum. The established relationship follows as:

$$
S_{\sigma}\left(\omega \mid H_{s}, T_{z}\right)=\left|H_{\sigma}(\omega \mid \theta)\right|^{2} \cdot S_{\eta}\left(\omega \mid H_{s}, T_{z}\right)
$$

The spectral moments of order $\mathrm{n}$ of the stress response, at zero vehicle speed condition for a given angle of heading, follows as:

$$
m_{n}=\int_{0}^{\infty} \omega^{n} S_{\sigma}\left(\omega \mid H_{s}, T_{z}, \theta\right) d \omega
$$

For the effect of directional spreading, the method considers confused short-crested sea condition. The formulation assumes a cosine squared spreading function, $(2 / \pi) \cos ^{2} \alpha$ with a spread from $-90^{0}$ to $+90^{\circ}$ on either side of the selected wave heading. The modified spectral moment appears as: 


$$
m_{n}=\int_{\theta-90}^{\theta+90}\left(\frac{2}{\pi}\right) \cos ^{2}(\alpha-\theta)\left(\int_{0}^{\infty} \omega^{n} S_{\sigma}\left(\omega \mid H_{s}, T_{z}, \alpha\right) d \omega\right) d \alpha
$$

where $\alpha$ is the spreading angle. The standard deviation of the stress process in a specific sea state follows as:

$$
\sigma=\left(m_{0}\right)^{1 / 2}
$$

\subsection{Spectral fatigue analysis}

Assuming the short-term wave induced bending stress response to be a narrow-banded Gaussian process, the peak value of stress $\mathrm{S}$ follows Rayleigh probability density function. The method obtains the Rayleigh probability distribution function $g(S)$, zero-up crossing frequency of the stress response $f$, and the bandwidth parameter $\varepsilon$ :

$$
\begin{aligned}
& g(S)=\frac{S}{4 \sigma^{2}} \exp \left[-\frac{S^{2}}{8 \sigma^{2}}\right] \\
& f=\frac{1}{2 \pi}\left(\frac{m_{2}}{m_{0}}\right)^{1 / 2} \\
& \varepsilon=\left(1-\frac{m_{2}^{2}}{m_{0} m_{4}}\right)^{1 / 2}
\end{aligned}
$$

The short-term damage incurred in the i-th sea state using an S-N curve of the form $\mathrm{N}=\mathrm{KS}^{-\mathrm{m}}[1]$ :

$$
D_{i}=\left(\frac{T}{K}\right) \int_{0}^{\infty} S^{m} f_{i} p_{i} g_{i} d S
$$

where,

$m, K=$ Physical parameters describing the S-N curve

$S=$ Stress range (twice the stress amplitude)

$f_{i} \quad=$ Zero-up-crossing frequency of the stress response in i-th sea state

$p_{i} \quad=$ Joint probability of $\mathrm{Tz}$ and $\mathrm{Hs}$ in i-th sea state

$g_{i} \quad=$ Rayleigh probability density function governing $\mathrm{S}$ i-th sea state

For calculating the damage ratio, the method uses the ABS bi-linear S-N curve. Details of the expression are given in reference [10], the closed form expression for damage is:

$$
D=\frac{T}{K}(2 \sqrt{2})^{m} \Gamma\left(\frac{m}{2}+1\right) \sum_{i} \lambda\left(m, \varepsilon_{i}\right) \mu_{i} f_{i} p_{i}\left(\sigma_{i}\right)^{m}
$$

The equation uses Wirsching's rain flow correction factor given by;

$$
\begin{gathered}
\lambda\left(m, \varepsilon_{i}\right)=a(m)+[1-a(m)]\left[1-\varepsilon_{i}\right]^{b(m)} \\
a(m)=0.926-0.033 m \\
b(m)=1.587 m-2.323
\end{gathered}
$$

$\mu_{i}$ Is the endurance factor having its value between 0 and 1 , gauges the involvement of the latter half to the damage incurred.

\section{Results and discussions}

\subsection{Hydrodynamic analysis.}


This study considers the midship section location of a simplified FPSO hull for spectral fatigue analysis. In order to obtain the influence of the vertical and horizontal bending moment on fatigue damage at different locations, the focus is on the stress responses at relatively more vulnerable locations due to global hull girder bending. Such locations identified are the longitudinal stiffener joints with the transverse bulkhead close to the midship section. Hydrodynamic analysis performed using Ansys Design Modeller, and Ansys AQWA [12] at zero speed condition gives the bending moment RAOs. Figure 2 shows the hydrodynamic model of the FPSO and Figure 3 shows the fourteen location points identified at the mid-ship section for the study. The analysis considers only half the midship section points because of the port and starboard symmetry. Table 2 gives the loading parameters for wave load computation on the hull. The hydrodynamic modelling requires mass distribution data inputs. Point mass inputs with associated mass moments of inertia represent the mass. The analysis gives short-term vertical and horizontal bending moment RAOs at the midship section for the two loading conditions namely, ballast and full load condition.

Table 2. Loading parameters

\begin{tabular}{|c|c|c|c|}
\hline Parameter & Range & Increment & Number \\
\hline Wave Frequency, $\omega$ & $0.1-2.0 \mathrm{rad} / \mathrm{s}$ & $0.05 \mathrm{rad} / \mathrm{s}$ & 39 \\
\hline Heading Angle, $\theta$ & $0^{\circ}-180^{\circ}$ & $22.5^{0}$ & 9 \\
\hline Vessel draught, $T$ & $16 \mathrm{~m}, 20 \mathrm{~m}$ & & 2 (Ballast, full load) \\
\hline
\end{tabular}

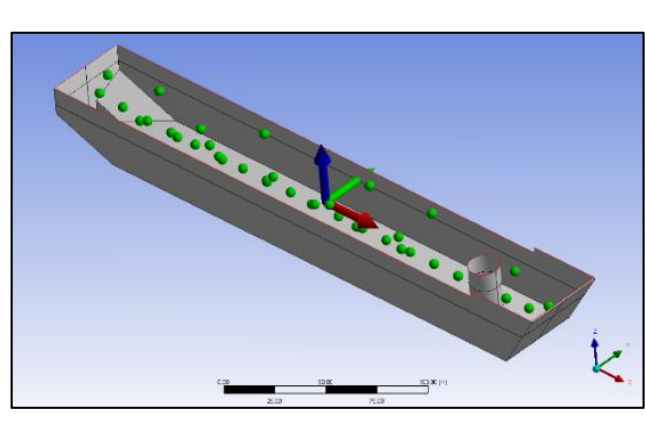

Fig. 2. Hydrodynamic model of FPSO for analysis in Ansys AQWA.

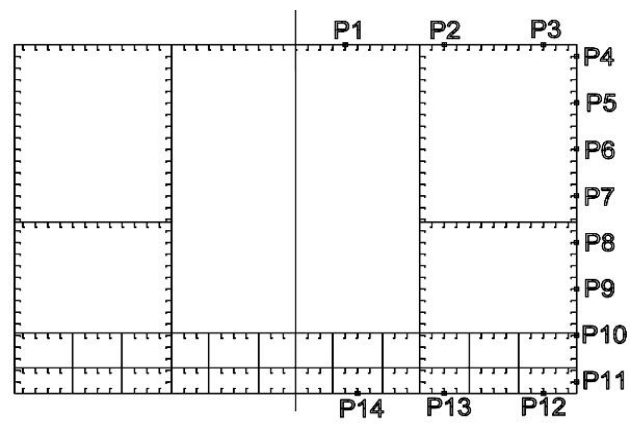

Fig. 3. Structural location points of interest at midship section

\subsection{Spectral fatigue analysis}

The primary step involves the determination of the stress transfer function and this, in turn, yields the PSDF of the stress response. A program coded in MATLAB performs the spectral fatigue analysis. Inputs to the code consist of the bending moment RAOs and geometrical parameters along with structural member location to compute the stress transfer function as shown in figure 4 , at the respective locations.

The program calculates the power spectral density function (PSDF) for the wave scatter diagram conforming to the ABS recommendation, to perform long-term statistical analysis. For short-term wave statistics, Pierson Moskowitz spectra define the wave spectrum for different sea states. The stress power spectral density function is computed using the stress transfer function. Further, the spectral moments are calculated based on the obtained stress PSDF as shown in figure 5, for calculating the fatigue damage.

This study has considered five sea states with significant wave heights ranging from 0.5 $\mathrm{m}$ to $4.5 \mathrm{~m}$ with an increment of $1 \mathrm{~m}$. For fatigue damage calculation this study uses the 
ABS bi-linear S-N curve. From the S-N curve data, the fatigue strength coefficients obtained are $4.227 \times 10^{13} M P a$ and $2.584 \times 10^{17} M P a$. The fatigue strength exponents are $m$ $=3$ and $m+\Delta m=5$.

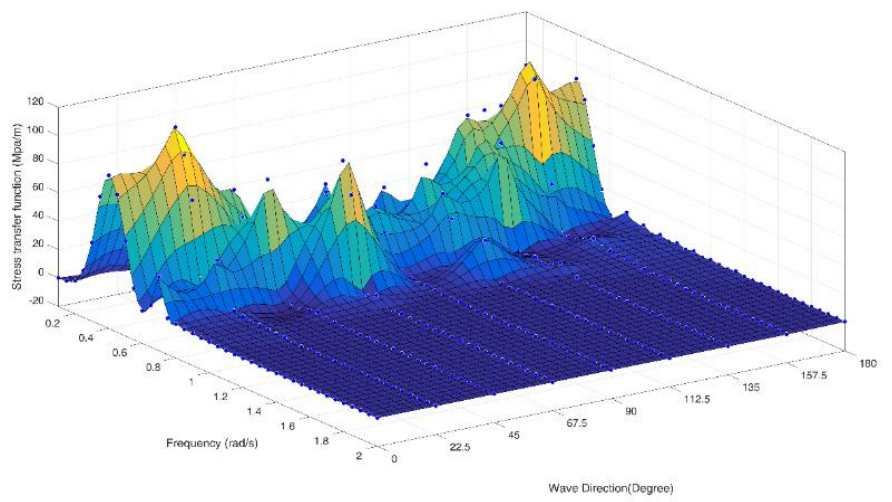

Fig. 4. Stress transfer function at location point 4 for $20 \mathrm{~m}$ draught (Sea State 5).

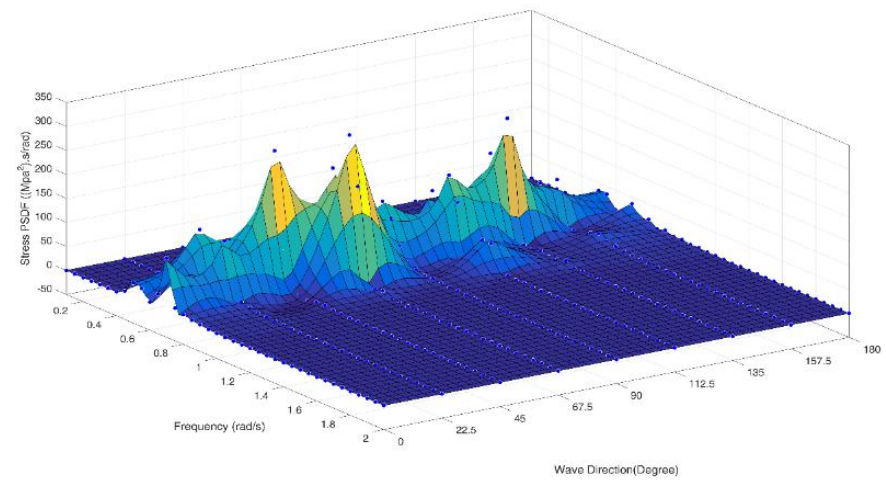

Fig. 5. PSDF of stress response at location point 4 for $20 \mathrm{~m}$ draught (Sea State 5).

The target fatigue life of the candidate FPSO considered as 20 years and performed the spectral fatigue analysis on the assumption that each loading conditions (i.e. the fully loaded and the ballast condition) will be for ten years. For the two loading conditions, i.e. draught $\mathrm{T}=20 \mathrm{~m}$ (fully loaded condition) and $\mathrm{T}=16 \mathrm{~m}$ (ballast condition), the method calculates the spectral fatigue damage ratio in all sea states. The fatigue damage ratios at location point 4 (point at the top of the side shell), during each sea state for both loading conditions is shown in table 3 . The individual damage ratios are further added together to obtain the combined damage ratio.

Table 3. Fatigue damage ratios at location point 4.

\begin{tabular}{|c|c|c|c|c|}
\hline $\begin{array}{c}\text { Sea } \\
\text { states }\end{array}$ & $H s(\mathrm{~m})$ & $\begin{array}{c}\text { The probability of } \\
\text { occurrence of } i^{\text {th }} \text { sea } \\
\text { state, } p i\end{array}$ & $\begin{array}{c}\text { Damage Ratio } \\
D i \text { at } T=20 \mathrm{~m}\end{array}$ & $\begin{array}{c}\text { Damage Ratio } \\
\text { Di at } T=16 \mathrm{~m}\end{array}$ \\
\hline S1 & 0.5 & 0.0215 & $1.238 \mathrm{e}-6$ & $9.954 \mathrm{e}-7$ \\
\hline S2 & 1.5 & 0.0535 & 0.0157 & 0.0126 \\
\hline S3 & 2.6 & 0.0325 & 0.2151 & 0.1729 \\
\hline S4 & 3.5 & 0.0133 & 0.2757 & 0.2215 \\
\hline S5 & 4.5 & 0.0047 & 0.2172 & 0.1750 \\
\hline
\end{tabular}


Calculated the fatigue damage ratio for both the loading conditions at all the chosen location points. For the study, three cases were considered namely, the effect purely due to vertical bending moment (PVB), the effect purely due to horizontal bending moment (PHB) and the combined effect due to the effect of vertical and horizontal bending moment (CVH). Figure 6 and 7 exhibit the damage incurred at each structural location for all the three cases for both fully loaded and ballast conditions.

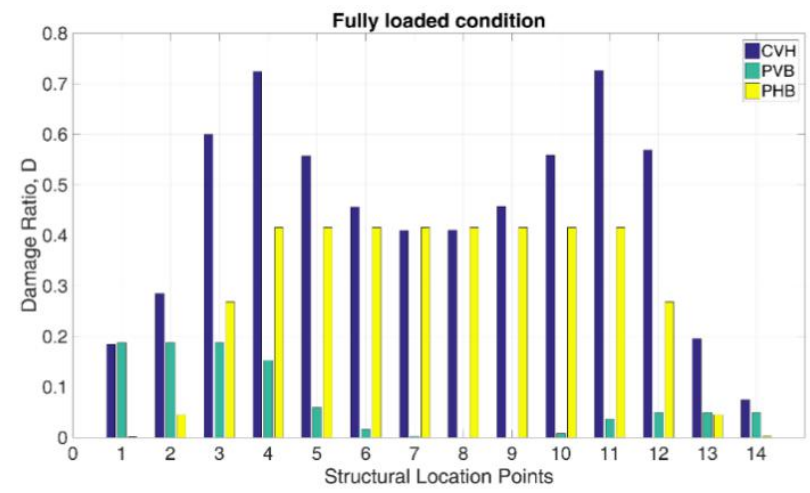

Fig. 6. Damage ratio at location points for the fully loaded condition.

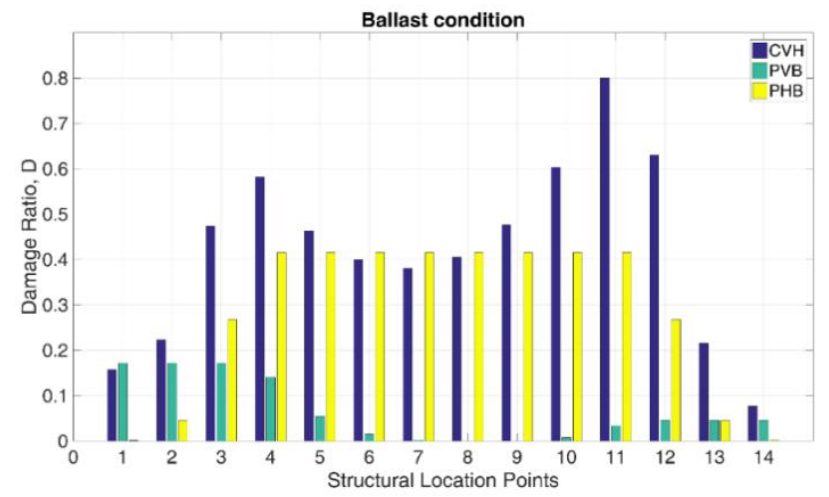

Fig. 7. Fatigue damage ratio at location points for ballast condition.

The results from the study show that for both the loading conditions, the fatigue damage ratio is maximum at the location points 4 and 11 on the side shell of the FPSO. For fully loaded condition the damage ratio at points 4 and 11 are 0.7242 and 0.7268 , respectively. For the ballast condition, the corresponding damage ratios at location points 4 and 11 are 0.5819 and 0.8003 , respectively. These regions must be given significant consideration during the design stage. Considering only the effect of an isolated vertical bending moment (green colour code in the Damage ratio graph) the location point 8 nearest to the plane of the neutral axis has negligible damage. The fatigue damage effect increases as the location move away from the neutral axis plane. The damage ratio at the location points in the deck region is 0.1871 for $T=20 \mathrm{~m}$ and 0.1718 for $T=16 \mathrm{~m}$, which is maximum in comparison with other points, as they locate further away from the neutral axis plane.

While considering the effect purely due to the action of the horizontal bending moment, a critical observation is that the fatigue damage effect incurred is identical in both the loading cases. The fatigue damage ratio at the location points 1 and 14 are 0.00096 at both draught conditions, which is minimum as they are nearer to the longitudinal centre plane and the value increases as the location move towards the side shell region. The damage ratio attains a maximum value of 0.4155 at the side shell region. 


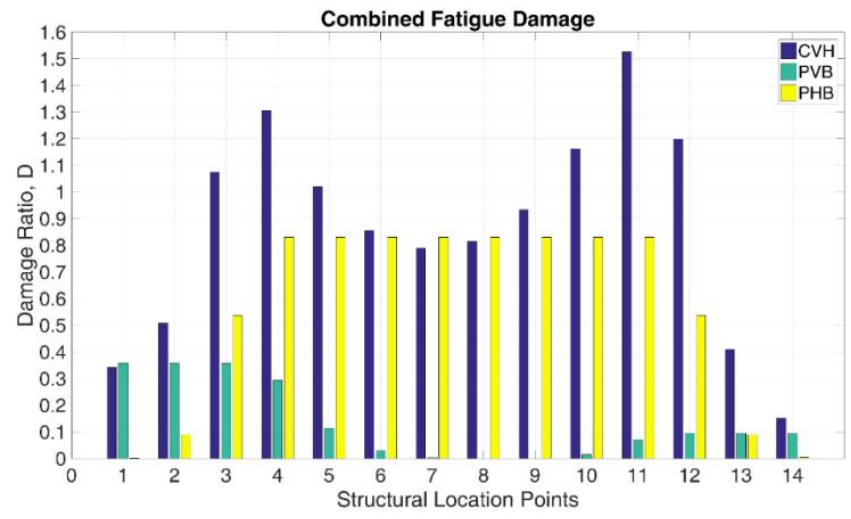

Fig. 8. Combined fatigue damage ratio at the structural location points

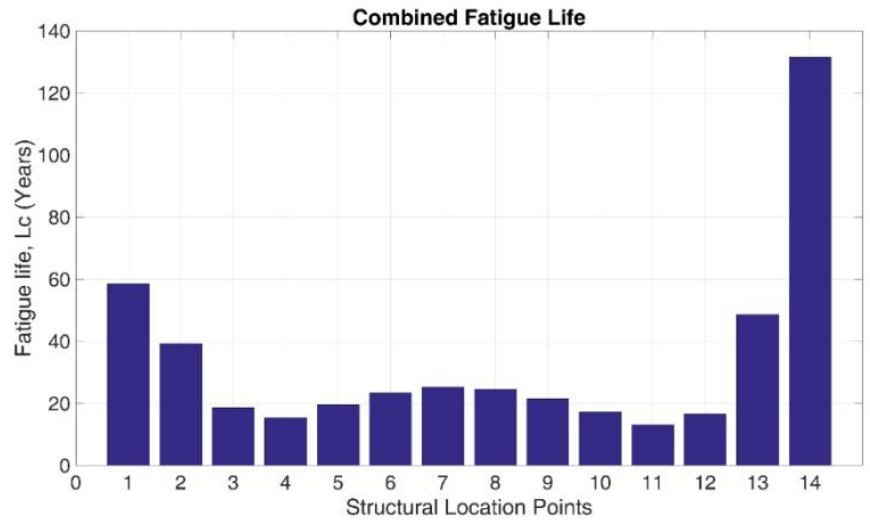

Fig. 9. Combined fatigue life at structural location points for case $\mathrm{CVH}$.

Figures 8 and 9 respectively depict the combined fatigue damage ratios and combined fatigue life obtained. Six structural locations namely 3, 4, 5, 10, 11 and 12 have damage ratios more than unity which indicates that the fatigue life at these locations is lower than the fatigue demand and the corresponding fatigue lives calculated are 18.62, 15.32, 19.60, $17.21,13.10$ and 16.67 years, respectively. By these results, it is recommended to have structural modifications to improve the midship section modulus with due considerations to the regions near the top and bottom areas at the side shell, for improving the fatigue life.

\section{Conclusion}

This study presents the variation of fatigue damage incurred in an FPSO vessel concerning the location of the structural member. Spectral fatigue analysis methodology was adopted and uses the Wirsching's rain flow correction factor. From the analysis results, the conclusions are as follows;

- The study confirms the general observation that structural members close to the top and bottom region of the side hull are more prone to fatigue damage.

- The fatigue damage due to the vertical bending moment is minimum at the structural locations near the neutral axis and maximum at the deck area. 
- The fatigue damage due the horizontal bending moment is identical in both loading cases. The damage ratio is maximum at the structural member locations on the side hull region and declines towards the centre line region.

\section{References}

1. A Gurney TR (1976). Fatigue design rules for welded steel joints. The welding Institute Research Bulletin, 17(5): 115-124.

2. Vughats JH, Kinra KR. 1976. Probabilistic fatigue analysis of fixed offshore structures, International Offshore Technology Conference, OTC Paper No. 2608

3. Kinra RK, Marshall PW. 1979. Fatigue analysis of the cognac platform, International Offshore Technology Conference, OTC Paper No. 3378

4. Xue J, Pittaluga A, Cervetto D. 1994. Fatigue damage calculation for oil tanker and container ship structures. Marine Structures 1994;7(6): 499-535.

5. Kukkanen Timo, Mikkola Timo PJ. 2004. Fatigue assessment by spectral approach for the ISSC comparative study of the hatch cover bearing pad. Marine structures; $17(1)$ : 75-90.

6. Lotsberg I, Cramer EH, Holtsmark G, Loseth R, Olaisen K, Valsgard 1997. Fatigue assessment of floating production vessel, In BOSS'97, Delft University of Technology, Delft, The Netherlands.

7. Lotsberg I, Nygard M, Thomas T. 1998. Fatigue of ship shaped production and storage units. International Offshore Technology Conference, Houston, TX, USA, OTC Paper No. 8775

8. Lotsberg I. 2001. An overview of the FPSO Fatigue capacity JIP, In OMAE, Rio de Janeiro, Brazil, OMAE01/MAT-3010

9. Wang YG. 2010. Spectral fatigue analysis of a ship structural detail - A practical case study. International Journal of Fatigue 32(2010) 310-317.Vughats JH, Kinra KR. 1976. Probabilistic fatigue analysis of fixed offshore structures, International Offshore Technology Conference, OTC Paper No. 2608

10. ABS 2010. Guide for spectral fatigue analysis for floating production, storage and offloading (FPSO) installation. American Beaureu of Shipping, Houston, USA

11. Rasmus Folso. 1998. Spectral fatigue damage calculation in the side shells of ships, with due account taken of the effect of alternating wet and dry areas. Marine Structures; 11:319-343.

12. ANSYS AQWA 2016. A potential flow-based analysis software for hydrodynamic analysis of ships, ANSYS Inc. Canonsburg, Pennsylvania, USA. 